\title{
Factores pedagógicos asociados con el compromiso de los universitarios con sus estudios
}

\author{
Lupe García-Cano ${ }^{1,2}$ y Pilar Colás-Bravo' \\ (1) Facultad de Ciencias de la Educación. Universidad de Sevilla. Sevilla - España. (correo-e: pcolas@us.es). \\ (2) Facultad de Ciencias Humanas y Sociales, Fundación Universitaria Los Libertadores. Bogotá - Colombia \\ (correo-e: Igarciac01@libertadores.edu.co).
}

Recibido Mar. 25, 2020; Aceptado May. 25, 2020; Versión final Jul. 3, 2020, Publicado Dic. 2020

\begin{abstract}
Resumen
El objetivo de este estudio es presentar la validación de una escala para registrar factores pedagógicos asociados al compromiso académico de los universitarios con sus estudios. La escala está compuesta por los siguientes factores: motivación, valores, oportunidades, estado emocional, engagement, gestión y estado anímico. La muestra estuvo conformada por 532 estudiantes, seleccionados mediante un muestreo aleatorio y estratificado. Se realizó un análisis factorial exploratorio (AFE) y un análisis factorial confirmatorio (AFC). Los resultados indicaron que los factores que conforman la escala explican el $70 \%$ de la varianza del constructo compromiso académico, además de mostrar una alta consistencia interna. El AFC confirmó la validez convergente y discriminante de la escala elaborada. Se concluye que la escala posee adecuadas propiedades psicométricas para valorar el compromiso académico de los estudiantes universitarios. Por tanto, puede ser de utilidad para diagnosticar situaciones y contextos universitarios que requieran de un mayor compromiso de los universitarios con su aprendizaje.
\end{abstract}

Palabras clave: implicación académica; educación superior; retención universitaria; análisis factorial exploratorio; análisis factorial confirmatorio

\section{Pedagogical factors that explain the engagement of university students with their studies}

\begin{abstract}
The objective of this research study was to validate a scale to record pedagogical factors related to academic engagement of university students and their studies. The scale was composed of seven factors: motivation, values, opportunities, emotional state, engagement, management, and mood. The sample was taken from 532 students selected through random and stratified sampling. An exploratory factor analysis and a confirmatory factor analysis were performed. The results indicated that the factors in the scale explained $70 \%$ of the variance of the academic engagement construct. In addition, these factors showed high internal consistency. The confirmatory factor analysis corroborated the convergent and discriminatory validity of the scale. It is concluded that the scale had adequate psychometric properties to assess academic engagement of university students. Therefore, the scale is useful to diagnose university situations and contexts that require increased university student engagement with their learning process.
\end{abstract}

Keywords: academic engagement; higher education; university retention; exploratory factor analysis; confirmatory factor analysis 


\section{INTRODUCCIÓN}

Uno de los indicadores de la calidad de los sistemas educativos es la tasa de éxito escolar, y como indicador negativo las tasas de abandono estudiantil. Esta temática por tanto es objeto de un corpus científico importante que trata, entre otros aspectos, de indagar en la comprensión y explicación de factores que inciden en uno u otra temática, ya que tiene repercusiones de naturaleza económica, social, nacional e internacional (Colás-Bravo, 2015; Wang y Brckalorenz, 2018). Colás-Bravo (2015) indica que la media europea de abandono escolar es del 16\%. Estudios económicos estiman que el fracaso universitario cuesta 5.772 euros por alumno al año (Goldenhersh et al., 2011). Estas valoraciones suponen un coste económico altísimo para los países, además de desaprovechamiento del capital intelectual y social que aportan los jóvenes universitarios. Las cifras en Colombia no son mejores, la retención universitaria es baja, pues uno de cada dos estudiantes que ingresa al sistema superior no culmina los estudios. Concretamente las estadísticas de deserción del sistema universitario colombiano se ubican entre el $45 \%$ y el 50\% (Ferreyra et al., 2017).

Entre los factores que afectan la retención universitaria, están los problemas financieros, la reputación y la calidad de la institución. Estos han sido estudiados en algunas aportaciones (Sembiring, 2015; Pike y Graunke, 2015, Roberts, 2018; Rodríguez et al., 2018). Pero, existe otra serie de factores asociados al estudiante. En este sentido Kahu y Nelson (2018) y Saito y Smith (2017), encontraron que el compromiso académico es un factor que influye en la culminación exitosa de los estudios universitarios; y que los estudiantes que se comprometen con sus estudios tienen más probabilidades de tener éxito académico. En esta línea, Shernoff et al., (2016), demostraron que el compromiso académico estudiantil correlaciona de manera positiva con el rendimiento académico, y una desconexión con los estudios conduce a un bajo desempeño académico, incluso hasta generar la desvinculación institucional.

En las últimas décadas, se ha dirigido una creciente atención hacia el compromiso académico estudiantil como un marco para comprender el fenómeno de la deserción, al menos en parte, debido a que este constructo es un aspecto cambiante e influenciable, entre otros, por los entornos de aprendizaje. Dada la importancia y repercusión de estas temáticas para las políticas universitarias y la mejora de los sistemas educativos en general, resulta de especial interés avanzar en la comprensión de estos fenómenos de forma científica. Uno de los retos para los investigadores es la delimitación y comprensión del constructo implicación en los estudios. Así como la identificación de factores asociados al mismo (Reyes-De Cózar, 2016). En estas coordenadas en donde se sitúa esta investigación que pretende validar un instrumento diseñado específicamente para registrar variables asociadas al compromiso académico. Este conocimiento y avance científico puede convertirse en una herramienta importante para mejorar los sistemas de enseñanza aprendizajes universitarios, así como ayudar al éxito académico de la población estudiantil universitaria.

\section{Hallazgos sobre el compromiso académico universitario}

La revisión de la literatura científica acerca del constructo engagement o compromiso académico saca a la luz numerosas aportaciones que iluminan sobre variables asociadas al mismo. Una de las primeras aportaciones se refiere a su conceptualización. Kuh (2003), describe el compromiso como tiempo, esfuerzo, intensidad y energía con la que los estudiantes se involucran en las actividades académicas y co-curriculares. Para Schiefele (2009) es la experiencia intensificada y simultánea de concentración, interés y disfrute. El interés por los estudios dirige la atención, refleja la motivación intrínseca, y estimula el deseo de continuar involucrado en una actividad. De otro lado, Saito y Smith (2017), encontraron que desde la perspectiva de un estudiante con bajo nivel de compromiso la educación es un obstáculo para socializar con sus compañeros, lo que lo lleva a invertir un esfuerzo mínimo en su trabajo escolar. Otros autores (Fredricks et al., 2016), identifican el compromiso académico como una construcción multifactorial que está relacionada con las prácticas pedagógicas y tiene un gran potencial como aspecto clave para la mejora institucional y los procesos de intervención educativa en el aula. Los factores que conforman el compromiso estudiantil, y que fueron abordados, en el presente estudio son: motivación, valores, estado de ánimo, estado emocional, oportunidades del aula y la gestión de los aprendizajes (Zhao y Kuh, 2004; Reyes-De Cózar, 2016).

La motivación de los estudiantes se ve afectada por numerosos aspectos de la cultura universitaria tales como mayor autonomía para su aprendizaje y gestión del tiempo, procesos de socialización, adaptación a una cultura académica diferente a los estudios previos, etc. La gestión de todos estos aspectos puede originar cambios en la motivación hacia los estudios. La motivación es un factor clave en el éxito o fracaso según la investigación científica (Lapointe y Perreault, 2013; Slanger et al., 2015).

Otro factor que incide en el compromiso académico es el valor. El bajo valor dado por el conocimiento, predice el esfuerzo mínimo de los estudiantes, poca concentración, indiferencia y el abandono de la actividad (Legault et al., 2006). Otros estudios confirman que, cuando los estudiantes valoran una actividad de aprendizaje en términos de alto valor de tarea, valor de utilidad, valor de interés, valor de logro, valor instrumental, metas 
futuras, consecuencias futuras, y perspectiva de tiempo futuro, la probabilidad que se comprometan con sus estudios es alta, lo que implica que persistan en un tema a lo largo del tiempo, y comprendan lo que están tratando de aprender.

Respecto al estado de ánimo, Schaufeli y Bakker (2004), definen el compromiso académico como un estado mental positivo, satisfactorio, persistente y penetrante, relacionado con las actividades 0 acciones emprendidas. Este se relaciona con el vigor, la dedicación y la absorción. El vigor, se caracteriza por altos niveles de energía y resistencia mental mientras trabaja, la voluntad de invertir esfuerzo en su trabajo, y la persistencia también frente a las dificultades. La dedicación, se caracteriza por un sentido de significación, orgullo, entusiasmo, desafío e inspiración. Y la absorción, que se caracteriza por estar totalmente abstraído y absorto en su trabajo, por lo que el tiempo trascurre con velocidad y uno tiene dificultades para desprenderse de los deberes.

Otros de los factores asociados a el compromiso académico estudiantil son el estado emocional, la gestión y las oportunidades de aula. El factor socioemocional, incluye emociones positivas y negativas que inciden en la realización de las actividades académicas del entorno escolar. Las emociones positivas predicen una regulación autónoma para el trabajo escolar, lo que a su vez predice un rendimiento académico positivo. De acuerdo con Kahu y Nelson (2018), las emociones son construcciones psicosociales que tienen una clara incidencia en el compromiso y el éxito académico. Cuando se les ofrece a los estudiantes oportunidades para satisfacer sus necesidades de autonomía, relación y competencia en la escuela, surge en ellos la ansiedad de participar, y al hacerlo, se desencadenan emociones energizantes como alegría, interés, curiosidad y entusiasmo por las tareas académicas que emprenden. Otro factor asociado es el contexto de aula o ambiente de aprendizaje. En este sentido se han identificado como dimensiones clave: Interacción, Exploración, Relevancia, Multimedia e Instrucción (Dunleavy y Milton, 2009).

Finalmente, la gestión hace referencia a las estrategias que despliega un estudiante para superar las dificultades. En esta línea las universidades han puesto en marcha diferentes estrategias y programas destinadas a ayudar al estudiantado y servir de acompañamiento con el fin de mejorar la retención universitaria, algunas son: implementación de tutorías de pares y mentorías académicas, oferta de seminarios de orientación; desarrollo de comunidades de aprendizaje, entre otras (Ferrer-Urbina et al., 2019). En todos estos hallazgos se fundamenta la escala de medida del compromiso académico elaborada, y su correspondiente validación, que se presenta en esta aportación. La inexistencia de escalas de esta naturaleza nos anima a presentar esta contribución que consideramos importante y relevante para la mejora de las enseñanzas universitarias y sus instituciones.

\section{METODOLOGÍA}

La metodología tiene un carácter cuantitativo, y el diseño de investigación es exploratorio. El estudio se realiza mediante la aplicación el Análisis Factorial Exploratorio (AFE) con SPSS y Análisis Factorial Confirmatorio (AFC) con AMOS v.24 a una escala previamente elaborada que recoge como principales dimensiones las aportaciones previas de la investigación científica disponible sobre factores asociados al implicación académica estudiantil o implicación académica.

\section{Participantes}

La población objeto de estudio son los estudiantes que cursan estudios universitarios en la Fundación Universitaria Los Libertadores de Bogotá (Colombia) Universidad durante el curso 2016. Se trata de lo que científicamente se denomina "población real". El tipo de muestreo es estratificado, en tanto se recaba información de los sujetos pertenecientes a las diferentes áreas de conocimiento en los que se engloba las titulaciones universitarias: Ciencias Administrativas y jurídicas; Artes, Humanidades y Ciencias Sociales e Ingeniería. Este muestreo se complementa con un muestreo aleatorio.

Se trabaja con un tamaño muestral que asume un error muestral del $5 \%$, y un nivel de confianza del $95 \%$. El tamaño muestral obtenido es de 532 sujetos obtenidos de forma aleatoria de una población de 10.276.

Del área de Ciencias Administrativas y Jurídicas, se encuestaron 162 estudiantes, lo que corresponde a un 30,5\%; del área de Artes, Humanidades y Ciencias Sociales 208 o 39,1\%, y del área de Ingeniería 162 o $30,5 \%$. El $53 \%$ de los estudiantes encuestados pertenecen al género femenino y el restante $47 \%$ al masculino. De otro lado, el $50,2 \%$ cursa sus estudios en la jornada diurna mientras el $49,6 \%$ en la nocturna. Finalmente, respecto a la caracterización de la muestra el $55 \%$ de los universitarios se encuentra laborando y el $45 \%$ se dedica a con exclusividad a sus estudios. 


\section{Instrumento}

El instrumento aplicado, fue una segunda versión de la Escala Multifactorial para la Medición del Engagement Educativo (EMMEE V1), diseñada y aplicada en la Tesis Doctoral de Reyes-De Cózar (2016) para estudiar y valorar el engagement o compromiso de los estudiantes de la Universidad de Sevilla - España. Los resultados obtenidos en esta investigación sirven de base para plantear una versión mejorada (V.2), y validarla en el contexto colombiano.

EI EMMEE - V.1 consta de 71 preguntas, distribuidas 6 escalas que miden los factores que inciden en el compromiso académico: la motivación, los valores, el estado emocional, las oportunidades del estudiante, y gestión de los aprendizajes y engagement. En este estudio se concluyó que este instrumento era susceptible de mejorar al replantear los ítems con baja fiabilidad y validez. Además, se planteó la pertinencia de aplicarlo en otros contextos universitarios, preferiblemente distintos al español, para aumentar el rigor y la transferibilidad de los resultados (Colás-Bravo et al., en prensa). Por ello, en el EMMEE, V.2., se modificaron aquellos ítems con valores de fiabilidad o validez bajos en la versión original del instrumento. En el EMMEE V.1, la opción de respuesta correspondía a una escala Likert clásica de 0-5, la cual se cambió en el EMMEE - V.2 por un rango de puntuación de 0 a 10 atendiendo a la recomendación de los autores, ya que en su estudio previo se concluyó que debido al rango pequeño de opción de respuestas se dificultó la obtención de una curva normal.

EI EMMEE V. 2., consta de 122 preguntas, distribuidas en 7 escalas. Cada escala, corresponde a uno de los factores que desde el punto de vista teórico inciden en el compromiso académico de los estudiantes universitarios. En este instrumento se recogen datos identificativos de la muestra relativos a: programa o titulación cursada, jornada académica, tiempo de permanencia en el programa como estudiante, edad y género. Estos datos se incluyen como apartado A del cuestionario. La escala B, corresponde al factor motivación y consta de 12 variables observables o ítems cuyo objetivo es descubrir los factores motivacionales que influyen sobre el compromiso académico de los estudiantes. La escala C, recoge 14 ítems referidos al sentido o valor otorgado por los estudiantes a su formación universitaria. La escala $D$, aborda los aspectos relacionados con los contextos de aprendizaje en el aula e incluye 20 variables observables. A diferencia de la escala de la V.1, en la V.2., se incluyen las oportunidades que brinda la universidad. La escala E, responde al factor emocional y la información se registra a través de un diferencial semántico que incluye términos que representan estados emocionales a través de una lista de adjetivos en forma bipolar, a través de 10 ítems. La escala $F$, identifica con 20 ítems el factor estrategias de gestión que usan los estudiantes a la hora de sobreponerse a las dificultades u obstáculos que se le presentan en su vida académica. La escala $G$ tiene como propósito determinar el tipo de engagement o compromiso de los estudiantes con sus estudios, a partir de 10 ítems. Finalmente, la escala H, es la nueva escala en la V.2. y consta de 17 ítems que miden el factor estado anímico de los estudiantes y está basada en la versión acortada (UWES-9) propuesta por Schaufeli y Bakker (2004).

\section{RESULTADOS}

Para dar respuesta a nuestras hipótesis de trabajo se utilizan de forma sucesiva dos procedimientos: el Análisis Factorial Exploratorio (AFE) con SPSS y Análisis Factorial Confirmatorio (AFC) con AMOS v.24.

\section{Análisis Factorial Exploratorio (AFE) con SPSS v.24}

Para determinar la adecuación de la matriz obtenida en el AFE se aplica el test Kaiser-Meyer-Olkin (KMO) y la Prueba de Esfericidad de Bartlett, con la base en los datos recogidos de 532 estudiantes y las variables observables distribuidas en las 7 escalas ya definidas. Las referencias a considerar para la valoración del índice KMO son: Si KMO $\geq 0.9$, el test es muy bueno; notable para $\mathrm{KMO} \geq 0.8$; mediano para $\mathrm{KMO} \geq 0.7$; bajo para $\mathrm{KMO} \geq 0.6$; y muy bajo para $\mathrm{KMO}<0.5$. Los resultados obtenidos se pueden ver en la Tabla 1.

Como se puede observar (Tabla 1) cuatro escalas tuvieron índice de KMO excelentes, tres valores buenos, y uno mediano $(0,787$ - muy cercano a 0,8$)$ lo que da fe de su adecuación muestral. También la prueba de esfericidad de Bartlett arroja valores de $x 2$ estadísticamente significativos en todas las escalas, $p<0.000$. Por tanto, rechazamos la hipótesis nula de ser una matriz de identidad, lo que indica que existen interrelaciones significativas entre los ítems del instrumento y por tanto resulta adecuado aplicar el análisis factorial. El segundo, fue la prueba de esfericidad de Bartlett, en el cual se encontró que el valor de significación para todas las escalas que conforman el instrumento de investigación fue .000, por ende, se podía aplicar el análisis factorial. Se nota que la varianza total explica por lo menos el $40 \%$ de cada factor, destacándose el valor de la escala $E(64,444 \%)$. En cuanto a la fiabilidad de consideraron como valores estimables un alfa de Cronbach entre 0,70 y 0,90 . Los resultados obtenidos se muestran en la Tabla 1. Como se observa, la fiabilidad global del instrumento es de 0.955 , lo que indica que esta versión tiene una excelente consistencia interna y fiabilidad. Todos los valores $\alpha$ de las escalas presentan coeficientes buenos y excelentes. Dado que las escalas con valor de Alfa aceptable son la B (.749) y la F (.761), se evaluó la posibilidad de eliminar algunos 
de sus ítems, con el fin de incrementar la fiabilidad de estas escalas. En el caso de la escala B, la eliminación de algún ítem no mejora la fiabilidad, pero en el caso de la escala F, eliminar los ítems 60, 69 y 79 incrementa la fiabilidad de .761 a .802, por lo cual podría ser aconsejable no considerar estos ítems para la aplicación definitiva del instrumento.

Tabla 1: Los índices de KMO y de esfericidad de Bartllett

\begin{tabular}{|c|c|c|c|c|c|c|}
\hline \multirow[t]{2}{*}{ Factores que conforman la escala EMMEE - V.2 } & \multirow[t]{2}{*}{ N ltems } & \multirow[t]{2}{*}{$K M O$} & \multicolumn{2}{|c|}{$\begin{array}{l}\text { Prueba de Esf. de } \\
\text { Bartlett }\end{array}$} & \multirow[t]{2}{*}{$\begin{array}{l}\text { V.2. Varianza } \\
\text { total explicada }\end{array}$} & \multirow[t]{2}{*}{$\begin{array}{l}\text { Alfa de } \\
\text { Cronbach }\end{array}$} \\
\hline & & & \begin{tabular}{|l} 
Chi-cuadrado \\
\end{tabular} & Sig, & & \\
\hline Factor Motivación / Escala B (BMOT) & 12 & 0,787 & 1372,992 & .000 & $40,679 \%$ & 0.749 \\
\hline Factor Valores / Escala C (CVAL) & 14 & 0,859 & 2338,747 & .000 & $42,006 \%$ & 0.816 \\
\hline $\begin{array}{l}\text { Factor Oportunidades / Escala D. } \\
\text { Oportunidades del estudiante (DOPA) } \\
\text { Oportunidades brindadas por la universidad (DOPAU) }\end{array}$ & 40 & 0,923 & 4094,200 & .000 & $45,027 \%$ & 0.921 \\
\hline Factor Estado Emocional / Escala E (EE) & 10 & 0.919 & 3603,133 & .000 & $64,644 \%$, & 0.931 \\
\hline Factor Gestión / Escala F (FGES) & 20 & 0.820 & 2979,730 & .000 & $43,389 \%$ & 0.761 \\
\hline Factor Engagement o compromiso / Escala G (GTIE) & 9 & 0,862 & 1466,456 & .000 & $42,308 \%$ & 0.802 \\
\hline Factor Estado de ánimo / Escala H (HEAN) & 17 & 0.909 & 3430,052 & .000 & $45,014 \%$ & 0.878 \\
\hline Global Escala EMDEE. & 122 & & & & & 0.955 \\
\hline
\end{tabular}

El AFE se realizó con el objetivo de eliminar las variables observables del compromiso académico que no tenían incidencia en la retención estudiantil universitaria. Después de 7 iteraciones en el programa SPSS 24 se obtuvo el mejor ajuste hallado del modelo. El AFE indicó que debían ser eliminados 89 de los 122 ítems que conformaban la escala EMDEE V2. En la tabla 2 se presentan los siete factores obtenidos y las variables que saturan cada factor. Como se puede notar en la tabla 2, la saturación de las variables observables es adecuada con los factores respectivos, excepto la variable 52, del factor estado emocional ya que quedó incluida en el factor de oportunidades.

Tabla 2: Variables que saturan cada factor de la escala EMMEE V.2

\begin{tabular}{|c|c|c|c|c|c|c|c|}
\hline & Factor & & & & & & \\
\hline & 1 & 2 & 3 & 4 & 5 & 6 & 7 \\
\hline BMOT9 & & & & & & & \begin{tabular}{|l|}
, 546 \\
\end{tabular} \\
\hline BMOT10 & & & & & & & ,698 \\
\hline BMOT12 & & & & & & & .435 \\
\hline CVAL15 & & 803 & & & & & \\
\hline CVAL18 & & ,481 & & & & & \\
\hline CVAL21 & &, 547 & & & & & \\
\hline CVAL22 & & 698 & & & & & \\
\hline CVAL23 & &, 517 & & & & & \\
\hline CVAL27 & &, 593 & & & & & \\
\hline DOPA37 &, 612 & & & & & & \\
\hline DOPA38 &, 482 & & & & & & \\
\hline DOPA39 &, 640 & & & & & & \\
\hline DOPA39U &, 406 & & & & & & \\
\hline DOPA40U & ,681 & & & & & & \\
\hline DOPA41 &, 834 & & & & & & \\
\hline DOPA48 & .464 & & & & & & \\
\hline DOPA48U &, 463 & & & & & & \\
\hline EEMO52 &, 614 & & & & & & \\
\hline EEMO53 & & & & & ,918 & & \\
\hline EEMO54 & & & & & ,767 & & \\
\hline EEMO59 & & & & & , 421 & & \\
\hline FGES67 & & &, 501 & & & & \\
\hline FGES75 & & & ,602 & & & & \\
\hline FGES77 & & & ,606 & & & & \\
\hline FGES78 & & & ,683 & & & & \\
\hline FGES79 & & & ,697 & & & & \\
\hline GTIEACA83 & & & & ,443 & & & \\
\hline GTIESOC87 & & & & ,769 & & & \\
\hline GTIEINT88 & & & & ,757 & & & \\
\hline GTIEINT89 & & & & ,664 & & & \\
\hline HEANVI99 & & & & & & ,713 & \\
\hline HEANDE103 & & & & & & ,846 & \\
\hline HEANAB104 & & & & & & \begin{tabular}{|l|}
.518 \\
\end{tabular} & \\
\hline $\begin{array}{l}\text { Método de ext } \\
\text { Método de rot }\end{array}$ & : fact & $\begin{array}{l}\text { zació } \\
\text { con n }\end{array}$ & $\begin{array}{l}\text { eje pr } \\
\text { alizaci }\end{array}$ & $\begin{array}{l}\text { pal. } \\
\text { Kaiser. }\end{array}$ & & & \\
\hline a. La rotación & vera & an 7 & ciones & & & & \\
\hline
\end{tabular}


Posteriormente, se procedió a eliminar las variables que no cumplían con las condiciones predeterminadas, para cada una de las escalas, con el método de extracción de máxima verosimilitud y rotación promax para minimizar el número de variables que tienen saturaciones altas en cada factor. Luego de los respectivos análisis, para algunas de las escalas se realizó de nuevo el procedimiento, hasta obtener un primer modelo teórico predictor de la retención universitaria. Los resultados por escala se presentan en la Tabla 3:

Tabla 3: AFE. Medidas de fiabilidad, validez discriminante y unidimensionalidad de los factores

\begin{tabular}{|l|l|l|l|l|l|}
\hline $\begin{array}{l}\text { Factores que } \\
\begin{array}{l}\text { conforman la escala } \\
\text { EMMEE - V.2 }\end{array}\end{array}$ & İtems incluidos en el AFC & KMO & \multicolumn{2}{|l|}{ Prueba de Esf. de Bartlett } & $\begin{array}{l}\text { VERSION 2. } \\
\text { Varianza total } \\
\text { explicada }\end{array}$ \\
\cline { 4 - 6 } & & & Chi-cuadrado & Sig, \\
\hline Motivación & $9,10,12$ & 0,754 & 1004,683 & .000 & $45,257 \%$ \\
\hline Valores & $15,18,21,22,23,27$ & 0,867 & 1111,465 & .000 & $47,738 \%$ \\
\hline Oportunidades & $37,38,39,39 U, 40 U, 41,48,48 U$ & 0,920 & 2941,713 & .000 & $51,072 \%$ \\
\hline Estado Emocional & $52,53,54,59$ & 0,919 & 3603,133 & .000 & $64,644 \%$, \\
\hline Gestión & $67,75,77,78,79$ & 0,788 & 2320,194 & .000 & $50,539 \%$ \\
\hline Engagement & $83,87,88,89$ & 0,774 & 843,282 & .000 & $61,126 \%$ \\
\hline Estado de ánimo & $99,103,104$ & 0,908 & 2866,376 & .000 & $48,178 \%$ \\
\hline
\end{tabular}

Cabe notar que el KMO para la mayoría de las escalas disminuye respecto a los valores iniciales presentados en la Tabla 1, (excepto para la escala C, pues al eliminar las variables observables 14, 17, 20, 24, 25 y 26 asciende de $\mathrm{KMO}=0,859$ a $\mathrm{KMO}=0,867$ ). Sin embargo, todos los valores de $\mathrm{KMO}$ presentan valores por encima de 0,7 . Contrario a lo anterior, el valor de la varianza total explicada para todos los factores asciende; es decir, se explica en mayor medida la relación entre compromiso escolar y retención. En cuanto a la escala E, el AFE muestra que todas las variables observables que la conforman explican adecuadamente el modelo, por ende, no se sugiere eliminar ninguna de estas. Ya con este modelo preliminar, conformado por los 33 ítems o variables observables relacionadas en las tablas 2 y 3 se procedió a realizar el Análisis Factorial Confirmatorio (AFC) con el programa AMOS.

\section{Análisis Factorial Confirmatorio (AFC) con AMOS}

En un primer momento, el AFE indicó que la variable observable 52, de la escala emocional quedó incluida en la de oportunidades. Y si bien algunos indicadores son adecuados como el RMSEA $(0,048)$ menor de la norma general de 0,08 , el Pclose mayor de $0,05(0,825)$, otros no lo son como el CFI que dio menor de 0,90. Además, todos los factores, exceptuando el estado emocional, no satisfacen la AVE dado que tienen valores por debajo de 0,50, incluso algunos por debajo de 0,40, por lo que se considera que son escalas que no son convergentes. Este estimativo motiva al mejoramiento del modelo. Sin embargo, con el mismo modelo se realizó la prueba de normalidad multivariante.

\section{Prueba de normalidad multivariante}

Con el fin de determinar los procedimientos a realizar para la estimación de los parámetros, es necesario identificar la forma de la distribución de los datos, específicamente, se valida si se cumple con la normalidad multivariante para las diferentes variables observables. Utilizando el programa AMOS, se encontró en la prueba de Mardia, un coeficiente de kurtosis de 444,866 con una región crítica de 104,625, por lo tanto, se incumple el supuesto; esto tiene incidencia en la elección del método de estimación de parámetros, el cual para el caso se sugiere el de Unweighted Least Squares Estimates - mínimos cuadrados no ponderados (ULS). Con lo anterior, se llegó al modelo presentado en la Figura 1. Se aprecia que el modelo usando el procedimiento de mínimos cuadrados no ponderados satisface los indicadores de bondad de ajuste. El RFI, AGFI y NFI todos superan los umbrales definidos para garantizar un buen ajuste, además, el coeficiente Standardized RMR = ,0439 menor de 0,08 satisface plenamente los requerimientos de un buen ajuste. Además, en la Figura 1, se nota que el modelo final consta de 24 variables observables de las 122 que conformaban la escala EMDEE V2 inicial, distribuidas en los 7 factores que inciden en el compromiso de los estudiantes universitarios.

En cuanto a las escalas se encontraron los resultados que se presentan en la Tabla 4. La fiabilidad compuesta supera en todos los casos el índice de 0,70. Con respecto a la AVE (Varianza media extraída), para muchos autores, se sugiere que el valor sea mayor de 0,5, no obstante, AVE es una medida estricta de validez convergente. El "AVE es una medida más conservadora que la CR. Basados en la CR solamente, el investigador podrá concluir si la validez convergente del constructo es adecuada, a pesar de que más del $50 \%$ de la varianza se debe al error" (Malhotra y Dash, 2011, p.702). En cuanto a la validez discriminante, se usa como criterio que los valores de la diagonal en la matriz (raíz cuadrada de AVE) sean mayores que las correlaciones de los diferentes factores. En el presente estudio se satisface plenamente dicha situación y, por lo tanto, se puede afirmar que las variables observables sólo aportan a su respectivo factor y no a otro de manera simultánea, por lo tanto, se cumple la validez discriminante. Lo anterior indica que el modelo se puede considerar adecuado. 


\section{Modelo de Medida Final \\ $\mathrm{RFI}=, 955$ \\ AGFI $=, 976$ \\ $\mathrm{NFI}=, 962$}

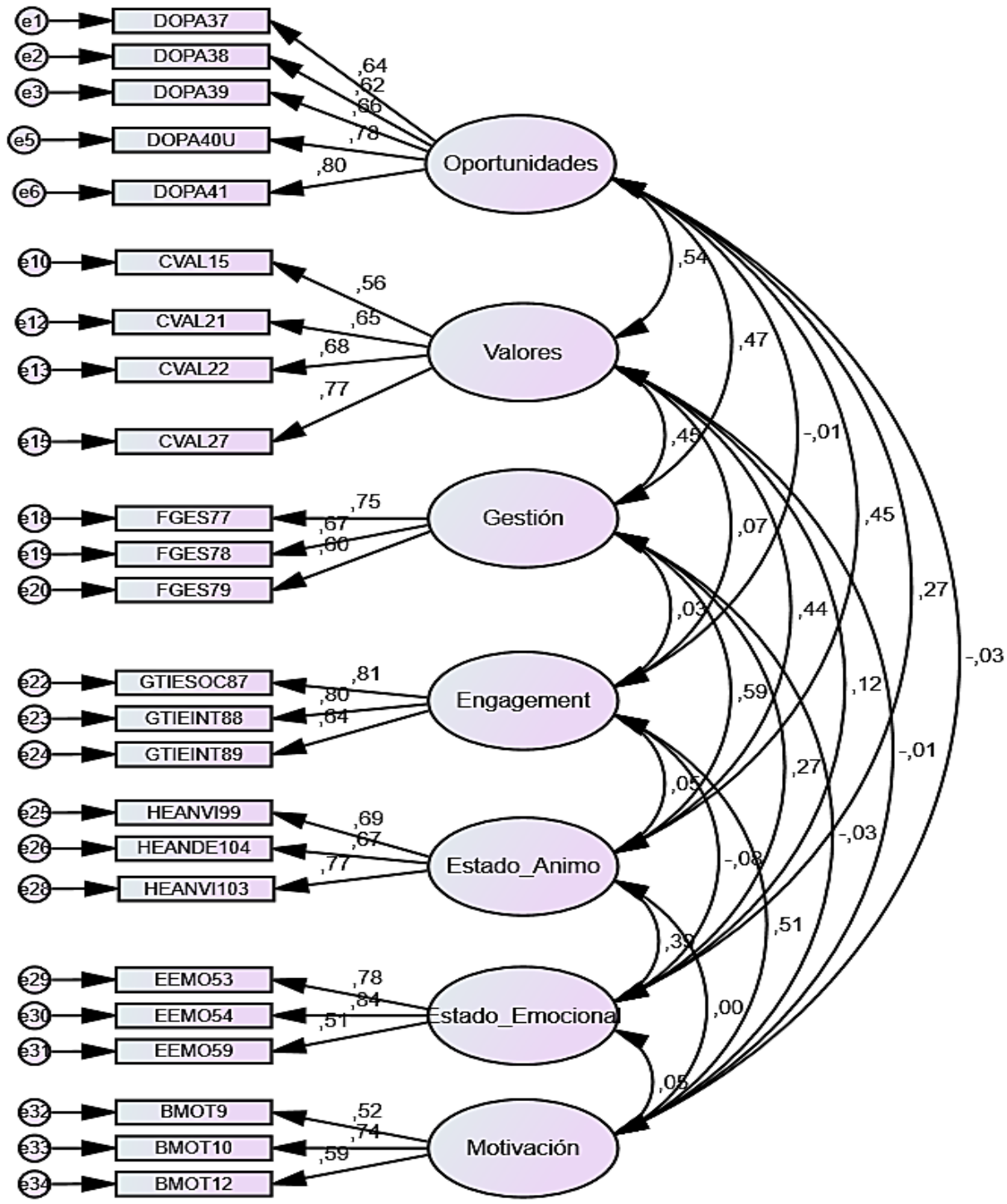

Fig. 1: Gráfico Path del AFC final procedimiento ULS

Tabla 4: Medidas de fiabilidad y validez discriminante modelo de medida final

\begin{tabular}{|c|c|c|c|c|c|c|c|c|c|}
\hline Factores & $\begin{array}{l}\text { Fiabilidad } \\
\text { Compuesta }\end{array}$ & AVE & Oportunidades & Valores & Gestión & $\begin{array}{l}\text { Compromiso o } \\
\text { engagement }\end{array}$ & $\begin{array}{l}\text { Estado } \\
\text { Ánimo }\end{array}$ & $\begin{array}{l}\text { Estado } \\
\text { Emocional }\end{array}$ & Motivación \\
\hline Oportunidades & 0,888 & 0,618 & 0,786 & & & & & & \\
\hline Valores & 0,824 & 0,541 & $0,569^{* \star *}$ & 0,736 & & & & & \\
\hline Gestión & 0,809 & 0,586 & $0,554^{* \star *}$ & $0,480^{* * *}$ & 0,766 & & & & \\
\hline $\begin{array}{l}\text { Compromiso o } \\
\text { engagement }\end{array}$ & 0,847 & 0,651 & $0,122^{* \star *}$ & $0,134^{* *}$ & $-0,003$ & 0,807 & & & \\
\hline Estado Ánimo & 0,714 & 0,457 & $0,770^{* \star *}$ & $0,463^{* * *}$ & $0,760^{* * *}$ & $0.145^{* *}$ & 0,676 & & \\
\hline $\begin{array}{l}\text { Estado } \\
\text { Emocional }\end{array}$ & 0,792 & 0,582 & $0,299^{* * *}$ & $0,088^{*}$ & $0,245^{* \star *}$ & $-0,11^{\star \star}$ & $0,513^{* *}$ & 0,763 & \\
\hline Motivación & 0,728 & 0,473 & $0,106^{*}$ & $0,221^{* * *}$ & 0,029 & $0,54^{* \star *}$ & 0,099 & 0,020 & 0,688 \\
\hline
\end{tabular}




\section{DISCUSIÓN}

La fiabilidad compuesta y la validez discriminante encontradas en el AFC, confirman que las escalas definidas previamente para los factores analizados, y las variables observadas son adecuadas. Lo anterior es un aporte importante a nivel científico, si se tiene en cuenta que luego de la revisión sobre el compromiso académico, no se cuenta con escalas con las fiabilidades encontradas. Otro hallazgo, es que el modelo de medida es adecuado, debido a que los indicadores AGFI, GFI, RFI y RMR, cumplen con los umbrales planteados (Peña et al., 2019). En el modelo obtenido, el AFC sugiere que para la escala B (motivación) las variables observables que determinan el compromiso académico son las referentes a la motivación intrínseca (ítems 9 , 10 y 12); es decir, que los universitarios se enganchan con sus deberes académicos cuando sienten que la educación es un medio para salir de su contexto, conocer lugares nuevos, interactuar con pares que tienen inquietudes parecidas, y pueden hacer uso de los recursos institucionales para desarrollar su capacidad crítica (Saeed y Zyngier, 2012; Rump et al., 2017).

Para el caso de la escala $\mathrm{C}$ - valor que le dan los universitarios a la educación, las variables que inciden en el compromiso académico son la 15, 21, 22 y 27; las cuales hacen referencia a si los estudios suponen para los estudiantes un medio de superación personal, y una forma de alcanzar la igualdad socio-económica (Legault et. al., 2006). Respecto a la escala D (oportunidades de aula), las variables observables 37 a 41, que implican en mayor medida a los universitarios con sus estudios tienen que ver con las acciones que realiza un profesor para resolver sus inquietudes, además de usarlas para ampliar los contenidos impartidos, y relacionarlas con el contexto en que ellos se desenvuelven. Por otra parte, el AFC indica que los estudiantes de educación terciaria se comprometen o afrontan satisfactoriamente sus estudios, cuando a nivel emocional se sienten optimistas, confiados y esperanzados, (Escala E - variables 53, 54 y 59), tal cual ha sido hallado en otros estudios (Kahu et al., 2017; García-Cano y Niño-Murcia, 2018).

De igual manera, el modelo obtenido indica que los estudiantes también se comprometen académicamente cuando cuentan con las herramientas de gestión necesarias para superar los obstáculos que les presentan (variables 77, 78 y 79). Estas variables indican que los individuos que implican en sus estudios cuando revisan y se apropian de los objetivos planteados en los distintos espacios académicos, además de pedir ayuda a los compañeros de clase y apoyarse en recursos externos como el internet cuando tienen dudas o no logran comprender los contenidos abordados durante las clases (Van Dinther et al., 2011; Goetez et al., 2013; Kahu, Stephens et al., 2015).

Finalmente, el modelo sugiere que los estudiantes universitarios que se levantan todas las mañanas con ánimo para ir al claustro universitario, estudian concentrados largos periodos de tiempo y consideran que los contenidos y estrategias planteados por los profesores en las aulas de clase son retadores, lo que genera compromiso o implicación con los estudios (variables 99, 103 y 104 - escala estado anímico). Por ende, son estos estudiantes quienes cuentan con la energía necesaria y suficiente para afrontar los estudios y culminarlos de manera exitosa; los concluyen o terminan a pesar de todos los obstáculos que se les presentan durante el transcurso de la carrera o titulación que decidieron cursar (Schaufeli y Bakker, 2004).

\section{CONCLUSIONES}

Con base en los resultados, podemos concluir que la escala "Escala Multifactorial para el Diagnóstico del Engagement Educativo (EMDEE) v2., es un instrumento válido para diagnosticar aspectos relacionados con el compromiso del alumnado universitario con sus estudios. Esta herramienta, puede resultar útil para caracterizar aspectos a mejorar y/o fortalecer en los sistemas universitarios con el fin de aumentar el nivel de compromiso de los estudiantes hacia sus estudios, y concluirlos exitosamente; es decir, persistir hasta alcanzar la titulación profesional.

De otro lado, los resultados de la medición, por parte de las instituciones universitarias, de las variables observables identificadas mediante el AFC como determinantes del compromiso de los universitarios en sus estudios, pueden permitir diseñar y poner en práctica estrategias y programas orientados a elevar el compromiso estudiantil, e incidir en la toma de medidas que contribuyan a aumentar la retención y, por ende, disminuir las tasas de abandono escolar. Por tanto, se recomienda el uso de este instrumento en otras poblaciones de habla hispana, y su respectiva validación para fortalecer el modelo teórico y la utilidad práctica del mismo.

\section{REFERENCIAS}

Colás-Bravo, P., El abandono universitario. Revista Fuentes, 16, 9-14 (2015).

Colás-Bravo, P., Reyes-De Cózar, S. y Conde-Jiménez, J., Validación de la escala multifactorial mixta de implicación académica educativo (EMMEE), Anales de psicología (en prensa). 
Dunleavy, J., y Milton, P., What did you do in school today? Exploring the concept of student engagement and its implications for teaching and learning in Canada, Canadian Education Association., Canada (2009).

Ferrer-Urbina, R., Karmelic-Pavlov, V., Beck-Fernández, H., y Valdivia-Pinto, R., Un modelo predictivo de fracaso/éxito académico a partir de indicadores de ingreso, en estudiantes de una Universidad Estatal del Norte de Chile, Interciencia, 44(1), 23-29 (2019).

Ferreyra, M.M., Botero, J., Avitabile, C., Haimovich, F., y otro autor., Momento decisivo: La educación superior en América Latina y el Caribe. Banco Internacional de Reconstrucción y Fomento/Banco Mundial (2017).

Fredricks, J.A., Filsecker, M., y Lawson, M.A., Student engagement, context, and adjustment: Addressing definitional, measurement, and methodological issues, https://doi.org/10.1016/j.learninstruc.2016.02.002, Learning and Instruction, 43, 1-4 (2016).

García-Cano, L., y Niño-Murcia, S., Percepciones sobre convivencia escolar y bullying en una institución educativa de Bogotá, http://dx.doi.org/10.17981/cultedusoc.9.1.2018.03, Cultura, Educación y Sociedad, 9(1), 45-58 (2018).

Goetez, T., Ludtke, O., Nett, U., Keller, M.M., y otro autor., Characteristics of teaching and students' emotions in the classroom: Investigating differences across domains, http://dx.doi.org/10.1016/j.cedpsych.2013.08.001, Contemporary Educational Psychology, 38(4), 383 - 394 (2013).

Goldenhersh, H., Coria, A., y Saino M., Deserción estudiantil: Desafíos de la universidad pública en un horizonte de inclusión, Revista Argentina de Educación Superior, 3(3), 96-120 (2011).

Kahu, E.R., Stephens, C.V., Leach, L., y Zepke, N., Linking academic emotions and student engagement: Mature-aged distance students' transition to university, https://doi.org/10.1080/0309877X.2014.895305, Journal of Further and Higher Education (39)4, 481-497 (2015).

Kahu, E., y Nelson, K., Student engagement in the educational interface: understanding the mechanisms of student success, https://doi.org/10.1080/07294360.2017.1344197, Higher Education Research and Development (37)1, 58-71 (2018).

Kahu, E., Nelson, K., y Picton, C., Student interest as a key driver of engagement for first year students, https://doi.org/10.5204/ssj.v8i2.379, Student Success 8(2), 55-66 (2017).

Kuh, G.D., What we're learning about student engagement from NSSE: Benchmarks for effective educational practices, https://doi.org/10.1080/00091380309604090, Change: The Magazine of Higher Learning, 35(2), 24-32 (2003).

Lapointe, M.C., y Perreault, S., Motivation: understanding leisure engagement and dis engagement, https://doi.org/10.1080/07053436.2013.836386, Loisir et Société / Society and Leisure, 36(2), 136-144 (2013).

Legault, L., Green-Demers, I., y Pelletier, L., Why do high school students lack motivation in the classroom? Toward an understanding of academic amotivation and the role of social support, Journal of Educational Psychology, 98, 567-582 (2006).

Malhotra, N.K., y Dash, S., Marketing Research an Applied Orientation. London: Pearson Publishing (2011).

Peña, P., González. C., y Montenegro, H., Desarrollo de una Escala de Prácticas y Concepciones de Evaluación de Profesores Universitarios (EPCEP), Formación Universitaria,12(6), 15-26 (2019).

Pike, G., y Graunke., S., Examining the Effects of Institutional and Cohort Characteristics on Retention Rates, Research in Higher Education 56(2), 146-165 (2015).

Reyes-De Cózar, S., "Fortalecer la implicación y el compromiso de los estudiantes con la universidad. Una visión multidimensional del engagement", Tesis doctoral, Dpto. Métodos de Investigación y Diagnóstico en Educación, Universidad de Sevilla, Sevilla-España (2016).

Roberts, J., Professional staff contributions to student retention and success in higher education, https://doi.org/10.1080/1360080X.2018.1428409, Journal of Higher Education Policy and Management, 40(2), 140-153 (2018).

Rodríguez, A.B., Espinoza, J., Ramírez. L., y Ganga, A., Deserción Universitaria: Nuevo Análisis Metodológico, http://dx.doi.org/10.4067/S0718-50062018000600107, Formación Universitaria,11(6), 107-118 (2018).

Rump, M., Esdar, W., y Wild, E., Individual differences in the effects of academic motivation on higher education students' intention to drop out, https://doi.org/10.1080/21568235.2017.1357481, European Journal of Higher Education, 7(4), 341355 (2017).

Saeed, S., y Zyngier, D., How Motivation Influences Student engagement: A Qualitative Case Study, http://dx.doi.org/10.5539/jel.v1n2p252, Journal of Education and Learning, 1(2), 252 - 267 (2012).

Saito, A., y Smith, M.E., Measurement and Analysis of Student (Dis) engagement in Higher Education: A Preliminary Study, https://doi.org/10.22492/ije.5.2.01, IAFOR Journal of Education, 5(2), 29-46 (2017).

Schaufeli, W. y Bakker, A., Job demands, job resources and their relationship with burnout and engagement: $A$ multi sample study. Journal of Organizational Behavior, 25, 293-315 (2004).

Schiefele, U., Situational and individual interest. In K. R. Wenzel y A. Wigfield (Eds.), Educational psychology handbook series. Handbook of motivation at school, 197-222 (2009). 
Sembiring, M., Student Satisfaction and Persistence: Imperative Features for Retention in Open and Distance Learning, https://doi.org/10.1108/ AAOUJ-10-01-2015-B002, Asian Association of Open Universities Journal, 10(1), 1-11 (2015).

Shernoff, D.J., Kelly, S., Tonks, S.M., Anderson, B., y otros tres autores., Student engagement as a function of environmental complexity in high school classrooms, https://doi.org/10.1016/j.learninstruc.2015.12.003, Learning and Instruction, 43, 52-60 (2016).

Slanger, W., Berg, E., Fisk., P., y Hanson, M., A longitudinal cohort study of student motivational factors related to academic success and retention using the College Student Inventory, Journal of College Student Retention: Research, Theory and Practice, 17(3), 278-302 (2015).

Van Dinther, M., Dochy, F., y Segers, M., Factors affecting students' self-efficacy in higher education, https://doi.org/10.1016/j.edurev.2010.10.003, Educational Research Review, 6(2), 95-108 (2011).

Wang, R., y Brckalorenz, A., Student engagement: An Exploration of Student and Faculty Perceptions, https://doi.org/10.5281/zenodo.1250402, Journal of International Student, 8(2), 1002-1033 (2018).

Zhao, C.M., y Kuh, G.D., Adding value: Learning communities and student engagement, Research in Higher Education, 45, 115-138 (2004). 